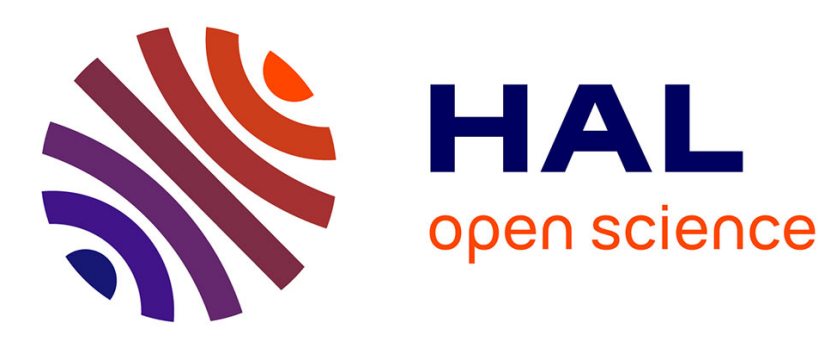

\title{
Modeling the Maintenance Time Considering the Experience of the Technicians
}

\author{
Hyunjong Shin, Kai-Wen Tien, Vittaldas Prabhu
}

\section{To cite this version:}

Hyunjong Shin, Kai-Wen Tien, Vittaldas Prabhu. Modeling the Maintenance Time Considering the Experience of the Technicians. IFIP International Conference on Advances in Production Management Systems (APMS), Sep 2019, Austin, TX, United States. pp.716-721, 10.1007/978-3-030-30000-5_87 . hal-02419198

\section{HAL Id: hal-02419198 \\ https://hal.inria.fr/hal-02419198}

Submitted on 19 Dec 2019

HAL is a multi-disciplinary open access archive for the deposit and dissemination of scientific research documents, whether they are published or not. The documents may come from teaching and research institutions in France or abroad, or from public or private research centers.
L'archive ouverte pluridisciplinaire HAL, est destinée au dépôt et à la diffusion de documents scientifiques de niveau recherche, publiés ou non, émanant des établissements d'enseignement et de recherche français ou étrangers, des laboratoires publics ou privés. 


\title{
Modeling the Maintenance Time considering the Experience of the Technicians
}

\author{
Hyunjong Shin ${ }^{1}$, Kai-wen Tien ${ }^{1}$, Vittaldas Prabhu ${ }^{1}$ \\ ${ }^{1}$ Pennsylvania State University, University Park PA 16802, USA \\ kut147@psu.edu
}

\begin{abstract}
Typically, maintaining a machine requires two different distinct tasks: to select which components to focus their attention; and the subsequent task is to check, repair or replace the selected components. For both tasks, the experience of technicians plays a critical role. An experienced technician is likely to select fewer components and requires less time for the subsequent task compared to an inexperienced technician. As a result, the maintenance time will be varied depending on the experience of the technicians. Extant research for maintenance has predominantly used exponential distribution family for modeling primarily because of its analytical tractability but at the cost of fidelity and inability to capture important characteristics such as technicians' experience. With the growing adoption of networked sensors based on Internet of Things (IoT), big data, and real-time machinery diagnostics using artificial intelligence it is imperative to develop models with better fidelity for maintenance operations. Therefore, in this paper, we explore a model based on using the negative-hyper geometric distribution for maintenance time that varies based on the technicians' experience. Our proposed approach requires more inputs such as (1) number of components, (2) number of components not in working state (3) technician's experience level, and (4) time to fix a component based on the technicians' experience. For instance, input for (2) could be obtained from IoT sensors and diagnostics. We study the efficacy of the proposed model using computer simulations and statistically characterize the possible impact of technician experience on the parameters of the maintenance distribution.
\end{abstract}

Keywords: Maintenance, Maintenance Time, Negative Hypergeometric Distribution, Technicians' Experience.

\section{Introduction}

Maintenance time consists of several steps: localize, isolate, adjust, disassemble, repair, interchange, reassemble, align, and checkout (return to normal condition) [1]. In many situations, especially for corrective maintenance, the process is composed of two different distinct tasks: to select which components to focus their attention; and the subsequent task is to check, repair or replace the selected components. For example, if technician removes the cover and finds out the selected component is in good condition, 
then he/she has to put the cover back and repeat the previous tasks until he/she successfully identify the problem. This process, which is similar to the trial-and-error process, contributes a large amount of time to the maintenance time and highly depends on technician experiences on fault localization (selecting which components to play).

How does experience impact the performance of maintenance? There are many different opinions in the literature. Cabahug et al. (2004) survey 105 respondents, including managers, training instructors, and directors in 2000. They consider 14 technician personal factors to examine technicians' proficiency. Their result shows up years of experience with equipment has a high correlation with technician proficiency [2]. Edwards et al. (2005) extend the research to 50 factors. They find that ten factors are significant for predicting maintenance proficiency including duration of training period and years of experience [3]. This study implies that experience is one of the instrumental factors in measuring technician proficiency. In Magne and Dag (2003), however, they claim that the experience has little impact on predicting problems in software maintenance. However, when they define technicians' experience, Magne and Dag only consider the number of years in general maintenance job but did not consider the time spent in maintaining the software [4].

With the growing adoption of networked sensors based on IoT, big data, and realtime machinery diagnostics using artificial intelligence, maintenance will be done more smartly even if a technician is inexperienced.

However, extant research for maintenance has perpetually lump-summed maintenance time and predominantly used the exponential distribution family such as exponential, gamma, phase-type and generalized-exponential distributions for modeling [1, 5-7]. It is primarily because of its analytical tractability. At the cost of fidelity, these models are, nevertheless, limited in ability to capture essential characteristics of maintenance such as technicians' experience. Based on our knowledge, no research had considered the experience of a technician when it modeled maintenance times. Hence it is inevitable to develop models with better fidelity for maintenance operations. Therefore, in this paper, we explore a model based on using the negative-hyper geometric distribution for maintenance time that varies based on the technicians' experience on the selecting task.

The rest of this paper is organized as follows. In section 2, we propose to model maintenance times using the Negative-Hyper Geometric distribution, which has the following inputs: (1) number of components, (2) number of components not in working state and (3) time to repair/replace a component. In section 3, we characterize the statistical properties of our model using computer simulation. Section 4 brings up our conclusions and possible works in the future.

\section{Modeling Maintenance Time}

Typically, maintaining a machine requires two different distinct tasks: (1) the first task is to select which components to focus their attention; and (2) the subsequent task is to check, repair or replace the selected components. The tasks repeat until the technician 
find all components that need to be repaired. In the model, we assume there are $N$ possible components which might cause a machine failure. The machine goes back to the working condition once all failed components are found and repaired. The time for the second type of task is varied by different failure types and components, so we model the time as a random variable $T_{i}$ (sub-task time), which is generally distributed, to describe the variation. In addition, we assume the operation time of the first type of task is negligible. Thus, the maintenance time $(M T)$, which consists of these two tasks, can be shown in equation (1)

Given that

$$
\mathrm{MT}=\sum_{i=1}^{X} T_{i}
$$

$T_{i}=$ time to check, repair and replace task $i$, is i.i.d. to $T_{1}$

$X=$ number of selected components to focus technicians' attention

\subsection{Modeling Component Selection using NHG Distribution}

Practically, selecting which components to focus attention is a similar process to the 'trial-and-error' process. The technician selects a component then check whether it requires replacement or repair. A technician takes necessary actions and then checks the next components until every component is in working state. This process also can be explained as drawing all 'fail' from a finite population without replacement in which each sample can be classified into two mutually exclusive categories: pass/fail. Therefore we formulated the number of selections, $X$, using the Negative Hyper-Geometric distribution, $X \sim N H G(N, M, k)$ [8]. In this model, we assume that all components must be in working state to properly maintain a machine. Otherwise, technicians continue to select more components, thus $k=M$. So, the distribution of $X$,

Where we define,

$$
\operatorname{Pr}(X=x)=\frac{\left(\begin{array}{l}
x-1 \\
k-1
\end{array}\right)\left(\begin{array}{l}
N-x \\
M-k
\end{array}\right)}{\left(\begin{array}{l}
N \\
M
\end{array}\right)}=\frac{\left(\begin{array}{l}
x-1 \\
M-1
\end{array}\right)}{\left(\begin{array}{l}
N \\
M
\end{array}\right)}
$$

$k$ is the number of components that need to be selected;

$M$ is the number of components which are not in working order;

$N$ is the number of all possible components which are not in working state and $M \leq N$.

\subsection{Modeling Experience Impact on Maintenance Time}

Two different experience effects can be defined in the model: (1) a more experienced technician requires a smaller number of selections in order to maintain the machine properly compared to an inexperienced technician; (2) a more experienced technician needs less time to finish each task in a maintenance process. In this paper, we discuss the first experience impact. We believe that this is because technicians can exclude 
some components in the first place based on their experience. Thus, in order to formulate this effect, we defined $N$ as $N^{*}$ which is

Where

$$
N^{*}=N-E E_{N}
$$

$N^{*}$ : number of possible components in technician's opinion

$E E_{N}$ : experience effect of $N, 0 \leq E E_{N} \leq N-M$

$E E_{N}$ can be expressed in constants or functions such as learning curves. However, it should be noted that the experience effect cannot be greater than $N-M$ since at least $M$ components should be checked even the technician is very talented and can find all failed components without any wrong selection.

\section{$3 \quad$ Model Inputs Analysis using Computer Simulation}

In this section, we analyze the sensitivity of the model's two inputs: $E E_{N}$ and $M$ to its variation by conducting a computational simulation. The simulation setting is:

- $\quad$ Set $N=20$ components and randomly assign $M$ failed components;

- Technician randomly identifies some components which they think it should be checked. All failed components must be identified.

- With experience effects $E E_{N}$, experienced technicians identify less components than inexperienced technicians

- A technician starts checking the components based on the order in step 2 . The sub-task time is constant and $T_{1}=10$.

- Maintenance time is collected after the technician repaired the last failed component

- 5000 maintenance times are collected (simulation iterations $=5000)$

\subsection{Input Analysis of $E E_{N}$}

$E E_{N}$ plays a critical role in this modeling. Although directly measuring $E E_{N}$ is very challenging, one could estimate $E E_{N}$ by reviewing a maintenance log. For example, one can obtain two information from the maintenance log: (1) the number of all possible components that need to be checked based on the failure mode, and (2) the number of all components that were checked by a technician. With this two information, we can fit the distribution to find out the $N^{*}$. Then we can estimate the $E E_{N}$ for the technician. However, even one can estimate the $E E_{N}$ from the $\log$, it is hard to estimate it accurately. Therefore, we simulated the model by only changing the variance of $E E_{N}$ to see how the variance of $E E_{N}$ affect the expected maintenance time. In this simulation, $M$ is set to be one. In addition, experience effects are followed by uniform distributions with three different intervals $E E_{N} \pm 1, E E_{N} \pm 2, E E_{N} \pm 3$.

Fig. 1 reveals us that the expected maintenance time is not biased by the variance of $E E_{N}$. In addition, the squared coefficient of variation (SCV) of maintenance time is increased as the variance of $E E_{N}$ is increased. Therefore, if we want to consider the second or higher moment of $M T, E E_{N}$ has to be carefully measured. 


\subsection{Input Analysis of $M$}

One common challenge of analyzing maintenance log is that it is almost impossible to have enough data for acquiring information about one failure mode. Since a machine can have multiple failure modes and the frequency of each failure mode is so small, it takes a significant amount of time to collect the data. Therefore, it would be good if a model can estimate the expected maintenance time of a machine regardless of failure modes. In order to estimate the expected maintenance time of a machine, the number of components that are not in working state is treated as a random number followed by uniform distributions with three different intervals $M \pm 1, M \pm 2, M \pm 3$. In this simulation, we set $E E_{N}=0$ and simulate model by changing $M$.

The results show in Fig. 2, we conclude that collecting data from different failure mode and using the average $M$ as the input of the model to calculate the mean and SCV of maintenance times is robust. Even the variation still impacts the values, the trend of the results is similar.
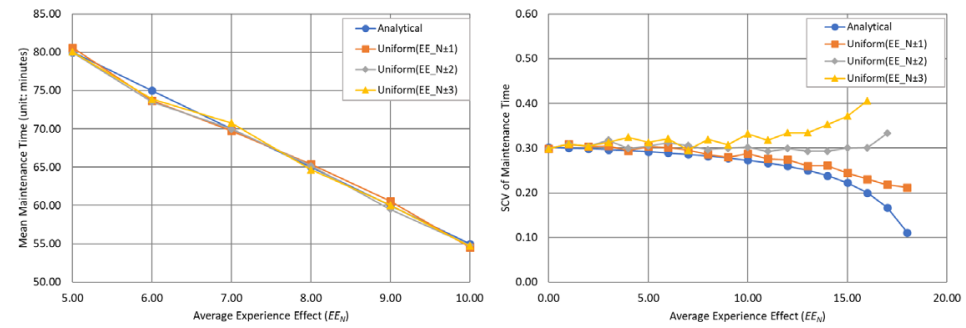

Fig. 1. Comparison of mean and SCV of maintenance time between analytical results and simulation results with uncertainty experience level
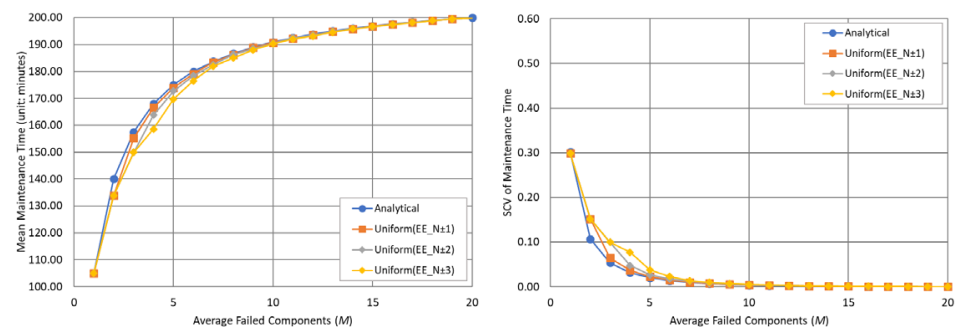

Fig. 2. Comparison of mean and SCV of maintenance time between analytical results and simulation results with multiple failure modes ( $M$ is uncertain)

\section{Conclusion and Future Research}

In this paper, we explore a model using the Negative Hyper-Geometric distribution for maintenance time that varies based on the technicians' experience. Our proposed approach requires inputs such as (1) number of components, (2) number of components not in working state (3) technician's experience level, and (4) time to fix a component. The reason to develop this model is to explore the impact of technicians' experience on 
maintenance times which will be primarily affected in the future due to the growth of usage of big data, machine learning, and A.I.

From the simulation results, we discover that the estimated expected maintenance time is robust to the variation of $E E_{N}$. The maintenance time doesn't get biased even when the uncertainty of $E E_{N}$ is increased. Therefore, we believe that estimating $E E_{N}$ from maintenance $\operatorname{logs}$ is one of the methods that can be used to estimate $E E_{N}$. In addition, we simulated that whether the model is robust to the variation of $M$. The simulation result reveals that the mean and SCV of maintenance time is robust when there is variation in $M$. Therefore, the model we proposed can be used to estimate the expect maintenance time of a machine in general. In sum, modeling maintenance time with Negative Hyper-Geometric distribution require more inputs than traditional modeling methods. However, this modeling method allows us to capture the technicians' experience effect and gives the power to analyze the average maintenance time.

In future work, we will validate the model with human experiment results. In addition, the model will be developed to assess the impact of IoTs and A.I. on maintenance decisions. Even though Negative Hypergeometric well describes the process of selecting components, the distribution is not well-known and not many analytical properties existed. Thus, we will try to develop approximation method for the model to increase its analytical tractability.

\section{Reference}

1. Dhillon, B. S. (2002). Engineering maintenance: a modern approach. CRC Press.

2. Cabahug, R. R., Edwards, D. J., \& Nicholas, J. (2004). Classifying plant operator maintenance proficiency: examining personal variables. Building Research \& Information, 32(2), 119-127.

3. Edwards, D. J., Yang, J., Cabahug, R., \& Love, P. E. (2005). Intelligence and maintenance proficiency: an examination of plant operators. Construction Innovation, 5(4), 243-254.

4. Jørgensen, M., \& Sjøberg, D. I. (2002). Impact of experience on maintenance skills. Journal of Software Maintenance and Evolution, 14(2), 123-146.

5. Dallery, Y. (1994). On modeling failure and repair times in stochastic models of manufacturing systems using generalized exponential distributions. Queueing Systems, 15(1-4), 199-209.

6. Liu, B., Cui, L., Wen, Y., \& Shen, J. (2015). A cold standby repairable system with working vacations and vacation interruption following Markovian arrival process. Reliability Engineering \& System Safety, 142, 1-8.

7. Liu, B., Cui, L., Wen, Y., \& Guo, F. (2016). A cold standby repairable system with the repairman having multiple vacations and operational, repair, and vacation times following phase-type distributions. Communications in Statistics-Theory and Methods, 45(4), 850-858.

8. Hu, D. P., Cui, Y. Q., \& Yin, A. H. (2013). An improved negative binomial approximation for negative hypergeometric distribution. In Applied Mechanics and Materials. Trans Tech Publications, Vol. 427, pp. 2549-2553. 